\title{
New insight into nanoparticle precipitation by electron beams in borosilicate glasses
}

\author{
M. M. Sabri ${ }^{1,2} \cdot$ G. Möbus ${ }^{1}$
}

Received: 10 January 2017/ Accepted: 23 May 2017/Published online: 31 May 2017

(c) The Author(s) 2017. This article is an open access publication

\begin{abstract}
Nanoprecipitation in different oxide glasses by means of electron irradiation in transmission electron microscopy (TEM) has been compared in this study. Upon irradiation, groups or patterns of nanoparticles with various morphologies and sizes were formed in borosilicate glasses, loaded with zinc, copper, and silver. The study successfully includes loading ranges for the target metal from doping level $(1 \%)$ over medium level $(20 \%)$ to majority phase $(60 \%)$. It is found that particle patterning resolution is affected by parallel processes of amorphous phase separation, glass ablation, and delocalised precipitation. In addition, via an in-situ study, it is confirmed that by heating alone without irradiation, no precipitate nanoparticles form.
\end{abstract}

\section{Introduction}

Glass-metal nanocomposite materials have recently attracted considerable interest due to their novel chemical and physical properties such as non-linear optical, nanoplasmonic, and quantum size effects [1-3]. Nobel metals such as gold and silver are amongst the most promising and most widely studied metallic nanoparticles in glass, while copper is sometimes included in extended studies [3]. In comparison with bulk metal, metallic

G. Möbus

g.moebus@sheffield.ac.uk

1 Department of Materials Science and Engineering, University of Sheffield, Sheffield, S1 3JD, UK

2 Present Address: Department of Physics, Faculty of Science and Health, Koya University, University Park,

Kurdistan Region, Iraq nanoparticles embedded in an insulating matrix reveal a wide range of useful physical features and provide excellent prospects for significant technological applications such as nanoplasmonic devices [4], memory switching devices [5], electrical charge storage [6], light emission [7], optical non-locality [8], optical devices [9], and also a common colorant for glasses [10].

The choice of oxide glass as a host matrix for precipitation experiments is because of the relative flexibility and homogeneity of the glass, allowing a large range of extra metal cation to be added. Especially alkali-borosilicate (ABS) glasses combine the advantages of low melting temperature with a large range of microstructures including various boron-oxide network models, possible amorphousamorphous phase separation, and tunable concentration of non-bridging oxygen (NBO), all properties having been exploited for both nanofabrication [11] and immobilisation of radionuclides [12].

A variety of methods have been used to prepare nanoparticles in oxide glasses, ranging from the standard annealing processes, or sol-gel preparation, over ion-exchange by diffusion, ion implantation, to irradiation with photon or particle beams [13-22]. Amongst the latter, laser irradiation $[23,24]$ is the most established technique in particular for the precipitation of copper and silver nanoparticles in glass. Hua et al. [25], for example, reported the space-selective precipitation of metallic copper and silver in $\mathrm{Cu}$ - and $\mathrm{Ag}$-doped silicate glasses using femtosecond laser irradiation. Under the irradiation, $\mathrm{Cu}$ and $\mathrm{Ag}$ ions capture the free electrons being created by multiphoton processes leading to photo-reduction. These atoms then aggregate to form nanoparticles during annealing.

Electron beam irradiation, however, is rarely reported for those glass-metal systems in spite of being a powerful 
technique for in-situ formation of different nanocrystals. Using transmission electron microscopy allows the nanocrystal formation to be observed live during fabrication [26-29] and mostly omitting any need for post-irradiation annealing [30]. In addition, the high energy of the electron beam has been exploited by researchers to modify the properties, structure, and composition of the glass [31]. Unusual micro/nanostructures or large size distribution of nanoparticles embedded in the glass can be obtained via this technique. Vostokov et al. [32] reported the capability of the electron beam irradiation in the formation of $2 \mathrm{D}$ metallic nanostructures in $\mathrm{Ag}$ and $\mathrm{Cu}$-doped glasses. Studies on the formation of silver nanoparticles of various diameters in glasses under low-energy electron beam irradiation have been reported by Nikonorov et al. [14, 32-35] and Brunov and co-workers [36]. These studies concentrate on general charge-induced surface aggregation observed ex situ by light microscopy without nanoscale imaging of the actual particle-enriched glass surface. Other metal nanoparticles explored via electron irradiation include bismuth [13] and germanium [37]. For the case of $\mathrm{Cu}$, only growth of pre-existing $\mathrm{Cu}$ nanoparticles under electron beam irradiation has been mentioned [38].

Upon specialising to electron beam irradiation-induced precipitation in oxide glasses, no publication is known to compare multiple metallic elements and multiple ranges of concentrations of the precipitating elements. At the same time, previous irradiation experiments used wide-beam non-local irradiation generating a homogeneous precipitation microstructure.

Differing from all the mentioned past work, we present in this study advances in the field of:

1. Irradiation experiments allow three-way comparison of three of the most promising candidate metals of $\mathrm{Ag}, \mathrm{Cu}$, and $\mathrm{Zn}$, which all are proven to be radiation sensitive and known to precipitate preferentially as metals rather than oxides. This choice includes the complete range from noble metal $(\mathrm{Ag})$, semi-noble metal $(\mathrm{Cu})$, to a typical reactive transition metal $(\mathrm{Zn})$. We also present the first comparison of glass compositions with largely varying concentration of the metal-target element, ranging from doping level to majority constituent.

2. Rather than irradiating with stationary beam, we explore the formation of patterns in glasses by spatially localised line scans generating metal nanoparticle areas separated by, otherwise, un-reacted glass matrix.

We further report on some observations about the influence of sample temperature (in-situ TEM heating) and on shape transformations of glass matrix observed as side effects during precipitation experiments.

\section{Experimental}

\subsection{Glass selection and preparation}

To represent the three envisaged concentration ranges for borosilicate glasses with extra metal ion loading for precipitation (high load of $>50 \%$, medium load of $\sim 20 \%$, and low load of $\sim 2 \%$ ), the 6 compositions in Table 1 have been chosen. They include the three target metals of $\mathrm{Ag}, \mathrm{Cu}$, and $\mathrm{Zn}$, and are labelled as Ag-BS, Cu-BS, and $\mathrm{Zn}-\mathrm{BS}$, respectively. Ideally, nine samples comprising three compositions each for all three elements would make up the complete study. However, Ag exceeds the glass-forming or solubility regions for the medium and (also for $\mathrm{Cu}$ ) high-load range; in fact, $\mathrm{Zn}$ is one of the few choices for a high-load experiment, as $\mathrm{ZnO}$ can act as network former together with borosilicate while also being (its own) nucleation promoter [39]. Low-load $\mathrm{Cu}$ glass (Cu-BS2) was melted and studied, in addition to the presented medium-load $\mathrm{Cu}-\mathrm{BS} 1$ and, however, did not precipitate under the electron beam, while a medium-load $\mathrm{Zn}$ glass (Zn-BS2) was found to behave very similar to the high-load Zn-BS1 [26], and, therefore, not presented here separately. The second silver-doped (Ag-BS2) glass was available from an earlier study for different motivations (vitrification of radionuclides [40]) and is used and reported here for comparison with the mainly used AgBS1 including some early line-scan patterning experiments.

Glass samples were produced by the conventional melting method using oxides, carbonates, and nitrides as raw materials. These include silica sand, cerium oxide, copper oxide, zinc oxide, boric acid, sodium carbonate, and silver nitrate. Then, these raw materials were mixed together to prepare glass batches of $300 \mathrm{~g}$. The melting temperatures have been kept similar for all glasses at $1400 \pm 50{ }^{\circ} \mathrm{C}$. Finally, the melt was fast quenched in water to obtain a fine glass frit to prevent nanoparticle precipitation during cooling.

\subsection{Sample preparation}

The glass frits were grinded manually into a fine powder in acetone using pestle and mortar. The mixture was then ultrasonicated for about 15 min to minimise the amount of agglomeration. Transmission electron microscope specimens were then prepared by picking up a small amount of the powder suspended in acetone using holey carbon film supported by either copper grids in $\mathrm{Ag}$ and $\mathrm{Zn}$ glasses or gold grid in $\mathrm{Cu}$ glass. 
Table 1 Chemical compositions of glass (mol\%)

\begin{tabular}{lllllllllll}
\hline Glass & \multicolumn{1}{l}{ Compositions $(\mathrm{mol} \%)$} \\
\cline { 2 - 10 } & $\mathrm{B}_{2} \mathrm{O}_{3}$ & $\mathrm{SiO}_{2}$ & $\mathrm{Na}_{2} \mathrm{O}$ & $\mathrm{K}_{2} \mathrm{O}$ & $\mathrm{CeO}_{2}$ & $\mathrm{Fe}_{2} \mathrm{O}_{3}$ & $\mathrm{Nd}_{2} \mathrm{O}_{3}$ & $\mathrm{CuO}$ & $\mathrm{Ag}_{2} \mathrm{O}$ & $\mathrm{ZnO}$ \\
\hline Ag-BS1 & 15 & 60 & 15 & 0 & 8 & 0 & 0 & 0 & 2 & 0 \\
$\mathrm{Ag}-\mathrm{BS} 2$ & 15 & 60 & 0 & 15 & 4 & 3 & 2 & 0 & 1 & 0 \\
$\mathrm{Cu}-\mathrm{BS} 1$ & 15 & 50 & 15 & 0 & 0 & 0 & 0 & 20 & 0 & 0 \\
$\mathrm{Cu}-\mathrm{BS} 2$ & 15 & 69 & 15 & 0 & 0 & 0 & 0 & 1 & 0 & 0 \\
Zn-BS1 & 20 & 20 & 0 & 0 & 0 & 0 & 0 & 0 & 0 & 60 \\
Zn-BS2 & 15 & 50 & 5 & 0 & 0 & 0 & 0 & 0 & 0 & 30 \\
\hline
\end{tabular}

\subsection{Transmission electron microscopy and irradiation technique}

In this study, a TEM of type JEOL JEM $3010 \mathrm{LaB}_{6}$ thermal filament at $300 \mathrm{kV}$ was generally used (except for Fig. 1c which is recorded at $200 \mathrm{kV}$ using a JEM 2010F). Our experiment is aimed as closely as possible to "in-situ" observations: the electron beam is used for both particle precipitation and for imaging. Intensity is varied by changing beam convergence/beam diameter, to switch between precipitation at high and imaging at low intensity. The majority of the experiments conducted here use "stationary irradiation" mode with electron beam diameters in excess of $100 \mathrm{~nm}$ to identify best conditions. Finally, line-scan irradiation mode using electron beams of below $100 \mathrm{~nm}$ in diameter is used to demonstrate patterning. The TEM is operated with the largest condenser aperture (CA) and spot size 1 as default with irradiation spread many times the camera diameter except where mentioned otherwise. These standard conditions correspond to $0.5-1 \mathrm{pA} / \mathrm{nm}^{2}$ current density. On one occasion (Fig. 2), no CA was inserted to achieve currents an order of magnitude higher than with the largest $\mathrm{CA}$, at the expense of less focusing capacity. In addition, the same TEM was used for in-situ heating experiments using a Gatan singletilt heating holder.

\section{Results}

Our findings, using examples from all three materials systems studied, are sorted by three overarching questions, following a brief introduction of stationary beam impact regions: (1) how can we classify impact regions containing new phases into amorphous-phase separation or particle precipitation? (2) what sort of line patterns can a moving electron beam leave behind, apart from or beyond the intended line of particles? and (3) how can the electron beam impact be separated from purely thermal trigger of precipitation of particles?

\subsection{Stationary irradiation}

Using stationary irradiation, impact crater regions can be generated and compared for the three glass types. All glasses share high beam sensitivity, with particle precipitation starting already at less than 1 min irradiation under the standard conditions; however, after prolonged irradiation, some important differences emerge. Particle size distribution being inversely proportional to the distance from the beam centre is the main characteristic for the $\mathrm{Zn}$ loaded glass Zn-BS2, Fig. 1a. The high Cu-glass Cu-BS1, Fig. 1b, shows an impact region centrally decorated with $\mathrm{Cu}$ particles while peripherally surrounded by 1 or 2 dark
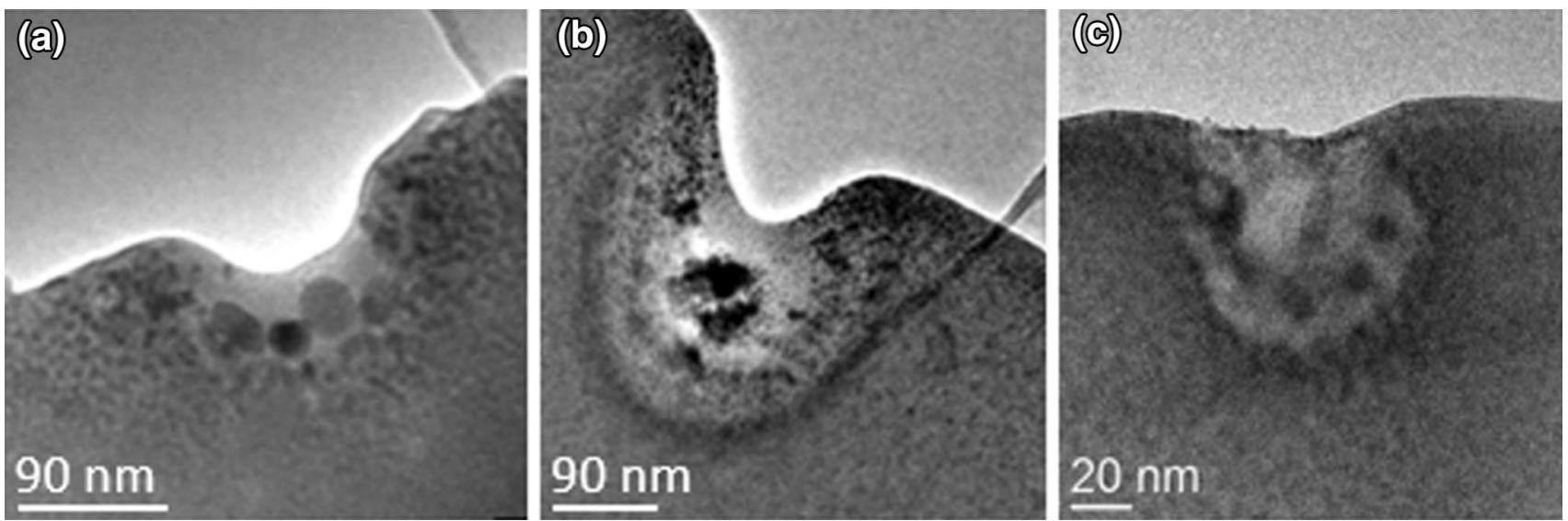

Fig. 1 Stationary electron beam impact craters for a $\mathrm{Zn}-$, b $\mathrm{Cu}-$, and $\mathbf{c}$ Ag-loaded glasses 
contrast rings, assumed to be rich in $\mathrm{Cu}$ clusters. Finally, the Ag-doped glass (Ag-BS2) shows a mixture of fine particles (near the sample edge) and larger dark patches in the thicker regions, Fig. 1c. The latter are diffuse rather than sharply bordered and too large to be Ag-rich only, which makes them most likely a phase separation effect, as shown in Sect. 3.2. Of interest is also the glass response to electron beam ablation, visible by the varying bright contrast, and the dent in the linear glass edge. The shape of the impact region is, therefore, crater-like. More details on stationary irradiation of our $\mathrm{Zn}$ and $\mathrm{Cu}$ glasses have been reported in [26, 41].

\subsection{Phase separation versus particle precipitation}

Using the $\mathrm{Ag} / \mathrm{Ce}$ glass (Ag-BS1) as an example, we now study the morphology of glass decomposition: while most generally, any decomposition could be labelled "glass phase separation", as it will generate new phases, we chose more restrictive notation: "phase separation" (PS) as amorphous glass-in-glass decomposition, such as of spinodal type, opposed to "nanoparticle precipitation" (NP) as separation of small distinct, mostly crystalline, particles as minority phase inside a (more or less changed) residual homogeneous majority phase. The main features for PS are: diffuse boundaries, similar volume fractions of both phases, and some characteristic length scale of separation ("wave length" if sinusoidal, related to diffusion length), while for NP we find: sharp abrupt boundaries between particles and glass matrix, large contrast changes, Bragg scattering contrast (if crystalline), distinct surface facets, and often symmetry-related particle shapes. The only inbetween case of small "droplet phase separation", sharing features of both PS and NP is not commonly observed in our samples.

Two interesting phenomena are compared in Fig. 2. On one hand, irradiating a rod-shaped glass fragment of the
Ag-BS1 glass by an electron beam of a diameter of about $500 \mathrm{~nm}$ for about $2 \mathrm{~min}$ at a high irradiation level resulted in precipitation of nanoparticles near the edge of the fragment and towards the centre of the beam, Fig. 2a. These nanoparticles are of about $3-10 \mathrm{~nm}$ in size and are circular in shape. This region is surrounded by another region of apparent amorphous phase separation (diffuse fringes) towards the thicker part of the fragment and along the outside of the circular electron beam impact, covering $1 / 3$ of the impact zone. Following an extra 2 min irradiation using the same conditions, the region of the amorphous phase separation has expanded (Fig. 2b), now covering $2 / 3$ of the impact zone. This enlarged PS zone now shows circular fringes of larger periodicity in Fig. $2 b$ compared to Fig. 2a, indicating longer diffusion range. Without crystallisation, grey scales can be interpreted as mass-thickness contrast and indicate that bright (dark) phases must contain low (high) atomic number elements. The nanoparticles on the glass edge vanished, while some larger ones further inside still persist with little growth. A later energy dispersive X-ray (EDX) chemical analysis measurement on another fragment of the same glass revealed that the composition change between regions consists of a roughly fourfold enrichment of both $\mathrm{Ag}$ and $\mathrm{Ce}$ in the dark regions compared to bright regions.

\subsection{Line-scan patterning: a comparison of $\mathrm{Zn}, \mathrm{Cu}$, and $\mathrm{Ag}$}

The aim of applying a moving electron beam across a glass region is for pattern formation, such that, e.g., metal-NP lines are fabricated. If continuous, these could become conductive interconnect lines on the nanoscale; otherwise, in the case of particle chains, they could serve as nanoplasmonic devices. Moreover, changes to glass reflectivity, refractive index patterns, light absorption, and diffraction by gratings are further prospective functional
Fig. 2 Originally homogeneous fragment of Ag-BS1 glass $\mathbf{a}$ after $2 \mathrm{~min}$ and $\mathbf{b}$ further 2 min irradiation, respectively
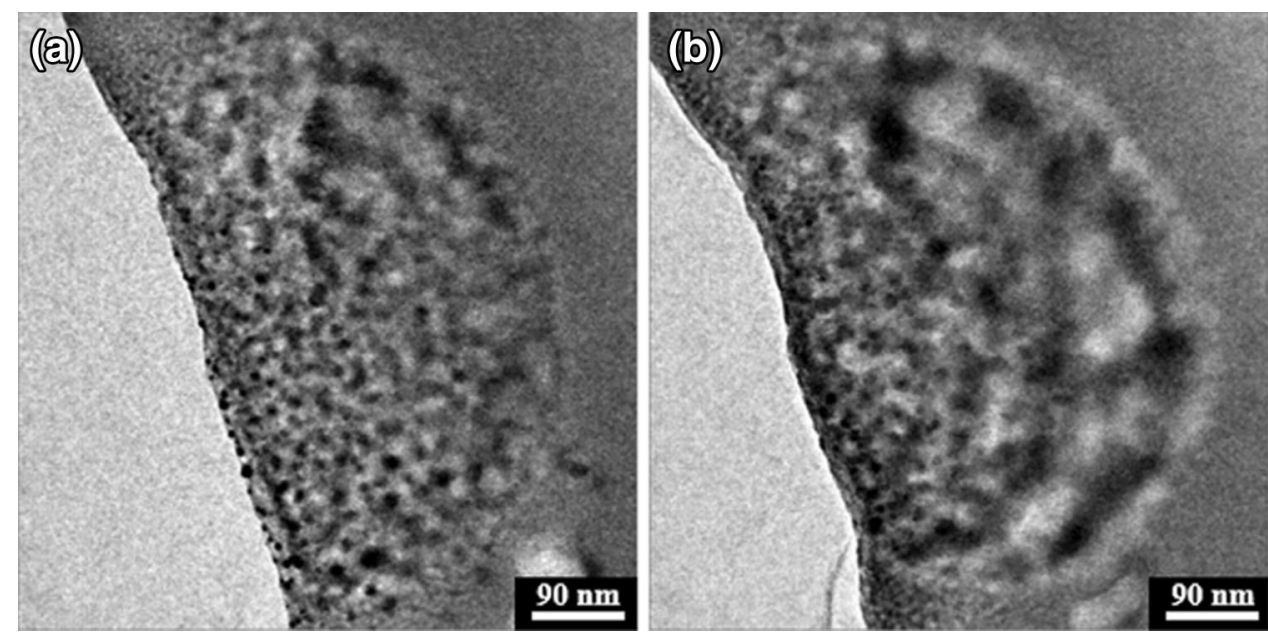
targets. We, therefore, explore the actual microstructural profile imprinted onto our glasses after line-scan patterning by the movement of a fixed electron beam through the glass material, here in comparison for all of our glasses containing $\mathrm{Zn}, \mathrm{Cu}$, and Ag. First, Fig. 3a shows a fragment of a high zinc borosilicate glass (Zn-BS) after performing linescan pattering. The scan involved ten steps of about 1-2 s exposure. This resulted in the formation of $\leq 10 \mathrm{~nm}$ sized spherical nanoparticles, the smaller the further away from the centre of the Gaussian electron beam. There are no continuous stripes and limited ablation.

Figure $3 \mathrm{~b}$ shows the line-scan pattering in a high copper-sodium borosilicate glass. A rod of this glass has been irradiated stepwise by e-beam movement with approximately $3 \mathrm{~s}$ dwell time. This resulted in formation of few about 2-3 nm sized nanoparticles inside the imprinted line of about $180 \mathrm{~nm}$ diameter. Identification of particles as mainly metallic $\mathrm{Cu}$ was confirmed in our previous work [41] through EELS and HRTEM. In addition, two peripheral black lines are produced on either side of the bright centre line as a result of this irradiation and this believed to be the accumulation of non-precipitated copper, migrated out of the centre line, which is the heaviest element in this glass.
Figure $3 c$, on the other hand, shows a line-scan pattering in silver/cerium-loaded sodium borosilicate glass (AgBS1). A rod of this glass has been irradiated with approximately $10 \mathrm{~s}$ dwell time to generate a structured line of about $400 \mathrm{~nm}$ length and about $90 \mathrm{~nm}$ total width. Three important details can be seen. First, electron beam irradiation resulted in the formation of circular $\sim 7 \mathrm{~nm}$ sized nanoparticles in two dis-continuous lines on either side of the e-beam centre. Second, e-beam-induced ablation of the glass matrix happened at the e-beam centre, and third, nanoparticles of well below $7 \mathrm{~nm}$ in diameter were found $>50 \mathrm{~nm}$ away from the electron beam centre. Further evidence can be gained from Fig. 3d, where an Ag-BS2 rod has been irradiated with moving beam across the rod diameter: round diffuse separation patches with separation length of around $20 \mathrm{~nm}$ are formed in the central beam path (analogue to Fig. 2), while smaller patches near the edge of the impact zone correspond to the tails of Gaussian beam distribution. Much smaller and relatively darker NP precipitates of below $5 \mathrm{~nm}$ size occur all across the impact zone with no apparent size sensitivity to the Gaussian beam intensity. Consequently, the NP might predominantly locate on the glass rod surface and also on the nearby carbon support film (likely due to evaporation
Fig. 3 TEM micrographs showing the process of line-scan patterning in our three glass systems loaded with a $\mathrm{Zn}, \mathbf{b} \mathrm{Cu}$, and $\mathbf{c}, \mathbf{d ~} \mathrm{Ag} / \mathrm{Ce}$ at two different magnifications
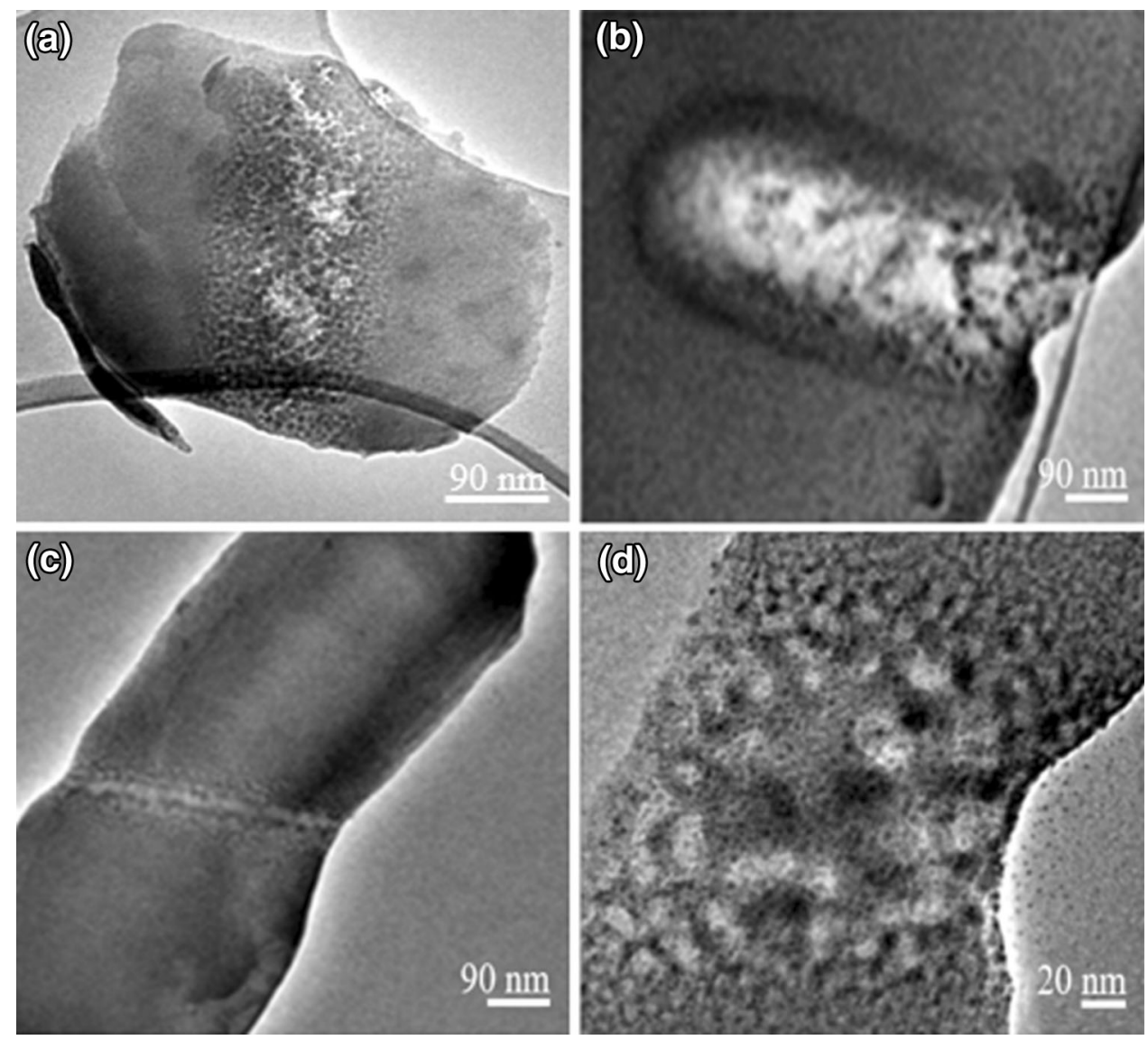
condensation), indicating that the particles form after the main phase separation event. An HRTEM image (not shown here) was obtained from the region of irradiation inside the rod including a typical silver nanoparticle with measured $d$-spacing of $0.202 \mathrm{~nm}$ for the (200) plane.

\subsection{Irradiation versus annealing for particle precipitation}

Using Zn-rich glass (Zn-BS) as our demonstrator, the effects of heating and irradiation are separated by the following strategy: first, particles are precipitated by room temperature irradiation for reference. Second, in-situ heating via TEM heating holder is applied. This will heat all sample glass fragments whether illuminated or not. Finally, a selected fragment is irradiated at high temperature and compared with non-irradiated nearby fragments.

A relatively homogeneous fragment of this glass (see Fig. 4a) in which no precipitates can be seen was successively irradiated in two steps, using intensities above the standard conditions with the beam focused to the field of view of the camera. First, after 2 min irradiation, the electron irradiation resulted in the precipitation of mostly roundish nanoparticles between 5 and $10 \mathrm{~nm}$ in size across the whole fragment, Fig. 4b. Second, after additional $2 \mathrm{~min}$ irradiation, the particles grow massively to $10-40 \mathrm{~nm}$ (see Fig. 4c), this time more faceted, some appearing hexagonal in projection. The residual glass fragment became highly electron-transparent indicative of the matrix losing most of its zinc by migration to particles. Our previous analysis of the precipitated nanoparticles via electron energy loss spectroscopy (EELS) and high-resolution transmission electron microscopy (HRTEM) revealed that these nanoparticles are in majority metallic [26].

The subsequent experiment on another fragment of the same glass, Fig. 5, includes three processes. First, irradiation of homogeneous (Fig. 5a) glass for about $5 \mathrm{~min}$ resulted in the precipitation of $10-50 \mathrm{~nm}$ sized nanoparticles (Fig. 5b). Due to irradiation with current density above the standard conditions, also a volume shrinkage and rounding of the overall glass fragment is observed, unlike in Fig. 4, motivated by radiation-induced fluidity [43], and to be further analysed in a forthcoming paper. Second, in-situ heating is applied over a range of temperatures inside the TEM, with Fig. 5c showing the result at $300{ }^{\circ} \mathrm{C}$. This heating of the already irradiated glass fragment did not (apart from few exceptions) result in the precipitation of new nanoparticles, but mainly in merging and growing of smaller particles (Ostwald ripening) to a size level rarely observe at room temperature. In addition, the overall morphology of the glass fragment did not change at these temperatures. Finally, a neighbouring glass fragment, Fig. 5d (upper left in the micrograph), subjected to the same heat treatment as the one from Fig. $5 \mathrm{c}$, but without any pre-irradiation, is confirmed to show neither any precipitation of nanoparticles nor changing of the morphology of the overall glass fragment.

\section{Discussion}

Of the six glass compositions from Table 1, three proved particularly successful in particle formation by stationary irradiation as well as pattern formation by line scan. However, achieved patterns differ in detail, mainly due to differing amounts of ablation, phase separation, and atomic migration appearing as non-intended alternative patterning phenomena. Amongst the three glasses, the $\mathrm{Zn}$-rich one proved most predictable: local precipitation was least affected by competing processes, and particle size best followed dose. This is linked to the discovery that $\mathrm{Zn}$ (being initially a $60 \%$ constituent as oxide) can be precipitated nearly completely leaving a rather stable binary borosilicate behind (very beam transparent). Next, the $\mathrm{Cu}$ glass proved unique as it had a tendency to produce ring patterns (Fig. 1) around an impact site for stationary
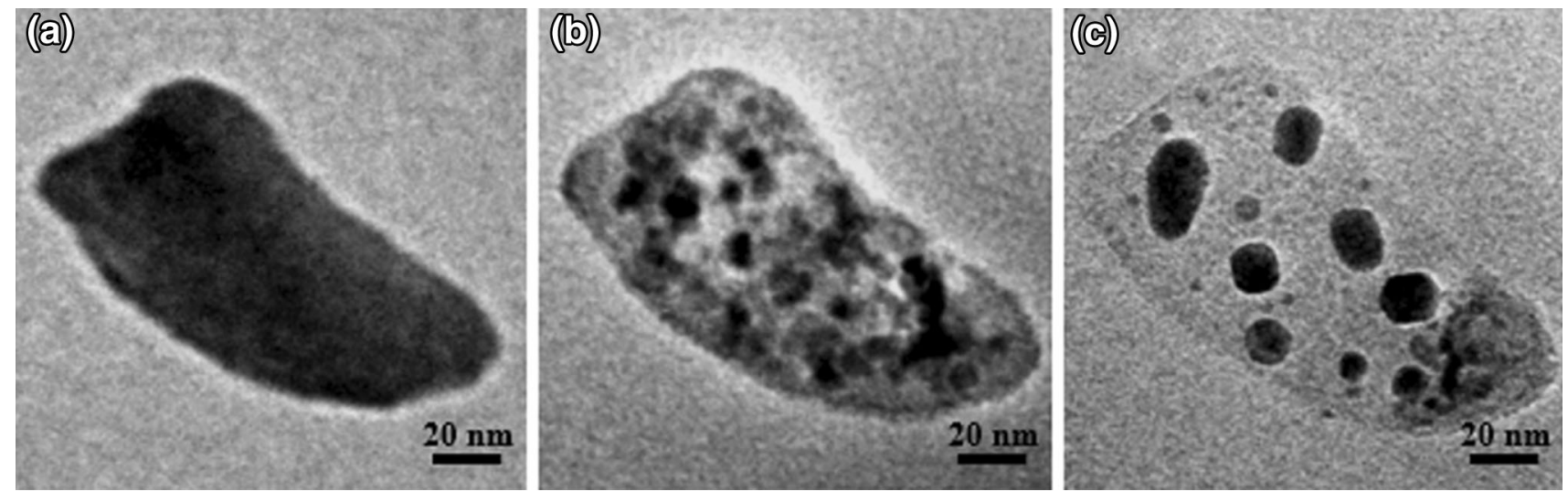

Fig. 4 High zinc borosilicate glass fragment, a prior to electron irradiation, $\mathbf{b}$ after irradiation for 2 min, and $\mathbf{c}$ for further 2 min 
Fig. 5 TEM images of a high zinc borosilicate glass fragment at a $0 \mathrm{~min}$, b $5 \mathrm{~min}$ of irradiation at high dose, $\mathbf{c}$ in-situ heating at $300{ }^{\circ} \mathrm{C}$ after irradiation, and $\mathbf{d}$ in-situ heating of the upper left glass fragment at $300{ }^{\circ} \mathrm{C}$ without irradiation
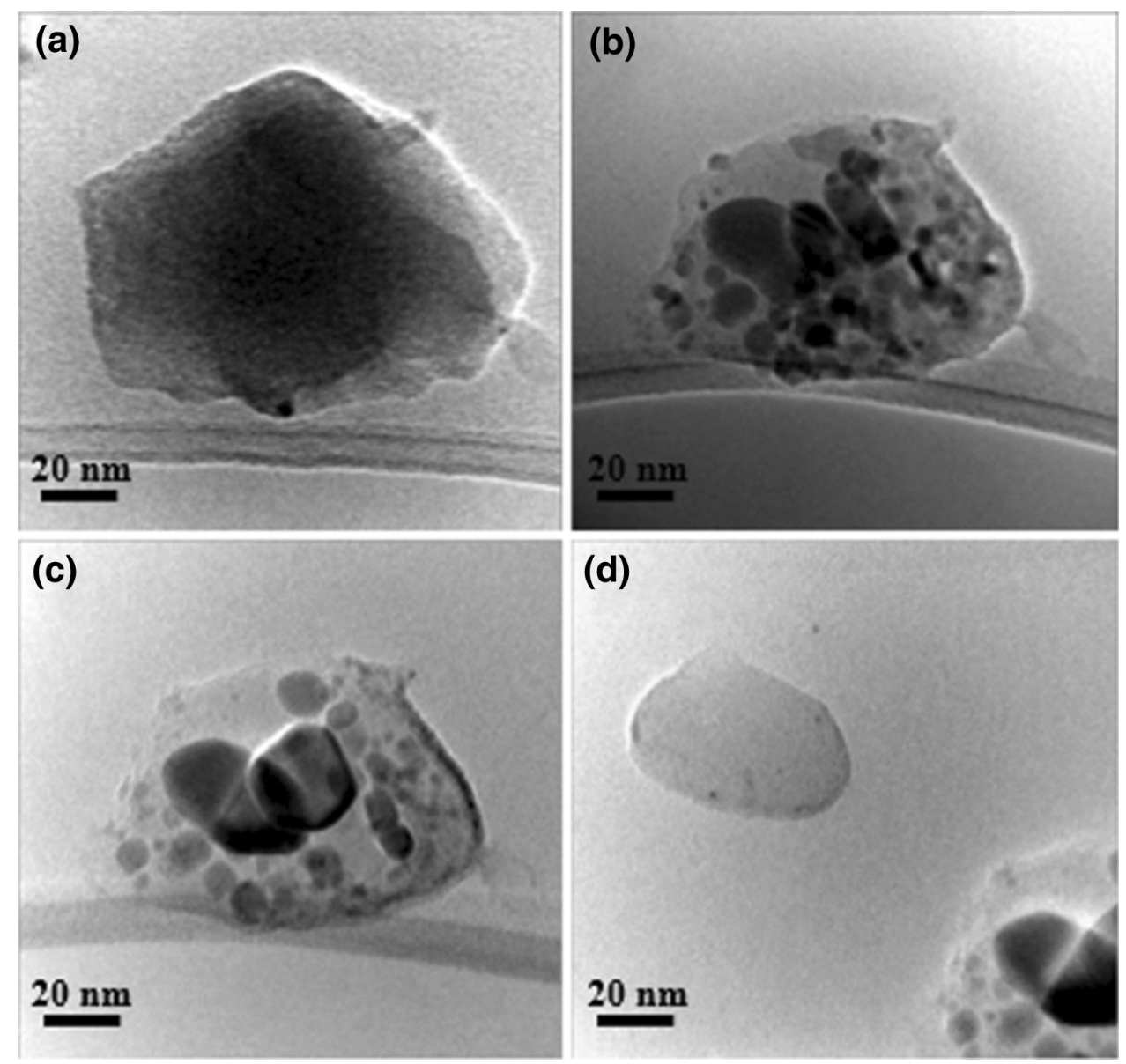

(d)

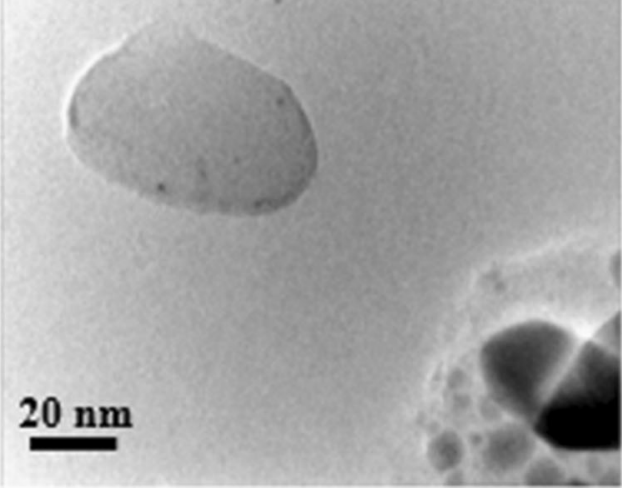

irradiation or double-stripe patterns (Fig. 3) in case of a line-scan, indicating $\mathrm{Cu}$ migration away from the beam centre before crystal nucleation, an observation not encountered with $\mathrm{Zn}$. This migration depletes the available metal in the centre line and counteracts predictability of particle-based patterning, although the complex $\mathrm{Cu}$ patterns might have interesting functional properties. Finally, the $\mathrm{Ag}-\mathrm{Ce}$-doped glass was unique in its tendency to have glass-in-glass phase separation accompanying particle formation giving rise to complex superposition patterns with Ag crystals appearing in parallel to diffuse dark regions rich in $\mathrm{Ag} / \mathrm{Ce}$.

Overall, scan speed, beam intensity, and diffusion length of the Me species in glass all combine to determine the final line profile made up of ablation, migration, and particle patterns. Pure ablative patterning [42] is another powerful nanostructure method, but should be avoided here. Ablation happened in all glasses, strongest in the $\mathrm{Cu}$ glass, where it was un-avoidable, while in the $\mathrm{Zn}$ glass, it could at least be minimised. Apart from the initial radiolytic liberation of metal atoms, irradiation-enhanced diffusion and irradiation-induced electric-field triggered atom migration [31] are the key contributing factors. The latter is believed to account for the $\mathrm{Cu}$ rings. It involves positive charging in the centre of the beam impact due to secondary electron emission. Radial electric-field lines then drive the positive ions out of the positively charged spherical zone. Beyond [31], the novelty of our $\mathrm{Cu}$ line-scan results lies in the on-the-fly reorganisation of multiple ring patterns during a beam line scan into two parallel lines which proves that migrated ions can be re-mobilised and swept out of the entire linear zone.

In comparison, in laser irradiation of glasses [24, 25] for particle precipitation, it is often assumed that the beam effect is through thermal annealing, except for special cases, where photo-reduction is the main effect [25]. In our case, however, our in-situ heating tests have confirmed that beam heating is not a contributing factor, and dwarfed by the pure irradiation-induced liberation of cations.

\section{Conclusions}

Generation of metallic nanoparticle regions on samples of a great variety of borosilicate glasses has been achieved by localised electron irradiation. Results confirm that 
patterning by metal precipitation is possible for the functional element being majority constituent or glass former (case of $\mathrm{Zn}$ ), medium constituent (case of $\mathrm{Cu}$ ), and minority dopant (case of $\mathrm{Ag}$ ). Demonstrations of line-scan irradiation modes support the feasibility of pattern generation; however, resulting line profiles are much more complex than just localising NP positions: the three effects of atom migration and glass ablation (main effect in $\mathrm{Cu}$ glass), glass phase separation (main effect in Ag-loaded glass), and particle precipitation (main effect in $\mathrm{Zn}$-loaded glass) are concurrent, overlapping, or competing, and need to be anticipated when selecting optimum combinations of dose rate, dwell time, and beam diameters. In addition, the study of in-situ heating in $\mathrm{Zn}$ glass confirmed that thermal effects alone do not result in precipitating nanoparticles, thereby proofing that radiation-induced precipitation by electrons is a separate physical effect, not emulating any other known annealing procedures.

Acknowledgements Our thanks go to the Iraqi Kurdistan Regional Government/Ministry of Higher Education and Scientific Research for the funding of the project as a part of Human Capacity Development Program (HCDP). The authors also thank Professor Russell J. Hand for his helpful contribution.

Open Access This article is distributed under the terms of the Creative Commons Attribution 4.0 International License (http://crea tivecommons.org/licenses/by/4.0/), which permits unrestricted use, distribution, and reproduction in any medium, provided you give appropriate credit to the original author(s) and the source, provide a link to the Creative Commons license, and indicate if changes were made.

\section{References}

1. F. Hache, D. Ricard, C. Flytzanis, Optical nonlinearities of small particles: surface-mediated resonance and quantum size effects. J. Opt. Soc. Am. 3, 1647 (1986)

2. M. Dragoman, D. Dragoman, Plasmonics, applications to nanoscale terahertz and optical devices. Prog. Quantum Electron. 32, 1 (2008)

3. L. Armelao, D. Barreca, G. Bottaro, A. Gasparotto, S. Gross, C. Maragno, E. Tondello, Recent trends on nanocomposites based on $\mathrm{Cu}, \mathrm{Ag}$ and $\mathrm{Au}$ clusters: a closer look. Coord. Chem. Rev. 250, 1294 (2006)

4. N.C. Lindquist, P. Nagpal, K.M. McPeak, D.J. Norris, S.H. Oh, Engineering metallic nanostructures for plasmonics and nanophotonics. Rep. Prog. Phys. 75, 063501 (2012)

5. D. Chakravorty, Memory switching in ion-exchanges oxide glasses. Appl. Phys. Lett. 24, 62 (1974)

6. A. Nakajima, T. Futatsugi, H. Nakao, T. Usuki, N. Horiguchi, N. Yokoyama, Microstructure and electrical properties of $\mathrm{Sn}$ nanocrystals in thin, thermally grown $\mathrm{SiO}_{2}$ layers formed via low energy ion implantation. J. Appl. Phys. 84, 1316 (1998)

7. J.V. Borany, R. Grötzschel, K.-H. Heinig, A. Markwitz, W. Matz, B. Schmidt, W. Skorupa, Multimodal impurity redistribution and nanocluster formation in Ge implanted silicon dioxide films. Appl. Phys. Lett. 71, 3215 (1997)
8. R. Chang, H.P. Chiang, P.T. Leung, D.P. Tsai, W.S. Tse, Nonlocal effects in the optical response of composite materials with metallic nanoparticles. Solid State Commun. 133, 315 (2005)

9. Y.H. Wang, S.J. Peng, J.D. Lu, R.W. Wang, Y.L. Mao, Y.G. Cheng, Optical properties of $\mathrm{Cu}$ and $\mathrm{Ag}$ nanoparticles synthesized in glass by ion implantation. Vacuum 83, 408 (2009)

10. H.D. Schreiber, M.A. Stone, A.M. Swink, Novel red-blue diachronic glass containing copper nanocrystals. J. Non-Cryst. Sol. 352, 534 (2006)

11. N. Jiang, Electron-beam fabrication of nanostructures in glasses. Microsc. Microanal. 16, 1660 (2010)

12. W.J. Weber, R.C. Ewing, C.A. Angell, G.W. Arnold, A.N. Cormack, J.M. Delaye, D.L. Griscom, L.W. Hobbs, A. Navrotsky, D.L. Price, A.M. Stoneham, M.C. Weinberg, Radiation effects in glasses used for immobilization of high-level waste and plutonium disposition. J. Mater. Res. 12, 1946 (1997)

13. S.P. Singh, B. Karmakar, In situ electron beam irradiated rapid growth of bismuth nanoparticles in bismuth-based glass dielectric at room temperature. J. Nanopart. Res. 13, 3599 (2011)

14. N. V. Nikonorov, I. A. Sidorov, A. V. Tsekhomskii, Silver nanoparticles in oxide glasses: technologies and properties, Silver Nanoparticles, ed. by D. P. Perez (In-Tech, Vukovar, 2010)

15. J. Zhong, W. Xiang, H. Zhao, W. Zhao, G. Chen, X. Liang, Synthesis, characterization and third-order nonlinear optical properties of copper quantum dots embedded in sodium borosilicate glass. J. Alloy. Comp. 537, 269 (2012)

16. S. Sirotkin, E. Cottancin, L. Saviot, E. Bernstein, A. Mermet, Growth of glass-embedded $\mathrm{Cu}$ nanoparticles: a low-frequency Raman scattering study. Phys. Rev. B. 85, 205435 (2012)

17. J. Zhang, J. Sheng, Formation and optical properties of copper nanoclusters in a silicate glass. Int. J. Hydro. Energ. 34, 3531 (2009)

18. E. Borsella, A.D. Vecchio, M.A. Garcia, C. Sada, F. Gonella, R. Polloni, A. Quaranta, L.J.G.W. Van Wilderen, Copper doping of silicate glasses by the ion-exchange technique: a photoluminescence spectroscopy study. J. Appl. Phys. 91, 90 (2002)

19. Y.H. Wang, C.Z. Jiang, X.H. Xiao, Y.G. Cheng, Third-order nonlinear optical response of $\mathrm{Cu} / \mathrm{Ag}$ nanoclusters by ion implantation under $1064 \mathrm{~nm}$ laser excitation. Phys. B 403, 2143 (2008)

20. A. Kipke, H. Hofmeister, Formation of silver nanoparticles in low-alkali borosilicate glass via silver oxide intermediates. Mater. Chem. Phys. 111, 254 (2008)

21. S.V. Serezhkina, L.T. Potapenko, Y.V. Bokshits, G.P. Shevchenko, V.V. Sviridov, Preparation of silver nanoparticles in oxide matrices derived by the sol-gel method. Glass Phys. Chem 29, 484 (2003)

22. F. Caccavale, G. De Marchi, F. Gonella, P. Mazzoldi, C. Meneghini, A. Quaranta, G.W. Arnold, G. Battaglin, G. Mattei, Irradiation-induced Ag-colloid formation in ion-exchanged sodalime glass. Nucl. Instru. Method. In Phys. Res. B. 96, 382 (1995)

23. Y. Teng, B. Qian, N. Jiang, Y. Liu, F. Luo, S. Ye, J. Zhou, B. Zhu, H. Zeng, J. Qiu, Light and heat driven precipitation of copper nanoparticles inside $\mathrm{Cu}^{2+}$-doped borate glasses. Chem. Phys. Lett. 485, 91 (2010)

24. S. Wackerow, A. Abdolvand, Generation of silver nanoparticles with controlled size and spatial distribution by pulsed laser irradiation of silver ion-doped glass. Opt. Exp. 22, 5076 (2014)

25. B. Hua, Y. Shimotsuma, M. Nishi, K. Miura, K. Hirao, Micromodification of metal-doped glasses by a femtosecond laser. J. Laser Micro/Nanoeng. 2, 36 (2007)

26. M.M. Sabri, R.J. Hand, G. Möbus, Zn nanodot patterning in borosilicate glasses by electron irradiation. J. Mater. Res. 30, 1914 (2015) 
27. N. Jiang, J. Qiu, J. Silcox, Precipitation of nanometer scale Zn crystalline particles in $\mathrm{ZnO}-\mathrm{B}_{2} \mathrm{O}_{3}-\mathrm{SiO}_{2}$ glass during electron irradiation. Appl. Phys. Lett. 77, 3956 (2000)

28. N. Jiang, B. Wu, J. Qiu, J.C.H. Spence, Precipitation of nanocrystals in glasses by electron irradiation: an alternative path to form glass ceramics? Appl. Phys. Lett. 90, 161909 (2007)

29. N. Jiang, J. Qiu, J.C.H. Spence, Precipitation of Ge nanoparticles from $\mathrm{GeO}_{2}$ glasses in transmission electron microscope. Appl. Phys. Lett. 86, 143112 (2005)

30. R. Espiau De Lamaestre, H. Bea, H. Bernas, J. Belloni, J.L. Marignier, Irradiation-induced $\mathrm{Ag}$ nanoclusters nucleation in silicate glasses: analogy with photography. Phys. Rev. B. 76, 205431 (2007)

31. N. Jiang, Damage mechanism in electron microscopy of insulating materials. J. Phys. D Appl. Phys. 46, 305502 (2013)

32. A.V. Vostokov, A.I. Ignat'ev, N.V. Nikonorov, O.A. Sidorov, A.I. Sidorov, A.V. Nashchekin, R.V. Sokolov, O.A. Usov, V.A. Tsekhomskii, Influence of electron beam irradiation on formation of silver nanoclusters in photothermorefractive glasses. Tech. Phys. Lett. 35, 35 (2009)

33. A.V. Vostokov, A.I. Ignat'ev, N.V. Nikonorov, O.A. Podsvirov, A.I. Sidorov, A.V. Nashchekin, R.V. Sokolov, O.A. Usov, V.A. Tsekhomskii, Effect of electron irradiation on the formation of silver nanoclusters in photothermorefractive glasses. Tech. Phys. Lett. 35, 812 (2009)

34. N.V. Nikonorov, A.I. Sidorov, V.A. Tsekhomskii, A.V. Nashchekin, O.A. Usov, O.A. Podsvirov, S.V. Poplevkin, Electronbeam modification of the near-surface layers of photosensitive glasses. Tech. Phys. Lett. 35, 309 (2009)
35. O.A. Usov, A.I. Sidorov, A.V. Nashchekin, O.A. Podsvirov, N.K. Kurbatova, V.A. Tsekhomskii, A.V. Vostokov, SPR of Ag nanoparticles in photothermochromic glasses. Proc. of SPIE. 7394, 73942J (2009)

36. V.S. Brunov, O.A. Podsvirov, A.I. Sidorov, D.V. Churaev, Formation of silver thin films and nanoparticles inside and on the surface of silver-containing glasses by electron irradiation. Tech. Phys. 59, 1215 (2014)

37. N. Jiang, J. Qiu, L.A. Gaeta, J. Silcox, Nanoscale modification of optical properties in $\mathrm{Ge}$-doped $\mathrm{SiO}_{2}$ glass by electron-beam irradiation. Appl. Phys. Lett. 80, 2005 (2002)

38. Y. Ito, H. Jain, D.B. Williams, Electron-beam induced growth of $\mathrm{Cu}$ nanoparticles in silica glass matrix. Appl. Phys. Lett. 75, 3793 (1999)

39. P. Taylor, D.G. Owen, Liquid immiscibility in the system $\mathrm{Na}_{2} \mathrm{O}$ $\mathrm{ZnO}-\mathrm{B}_{2} \mathrm{O}_{3}-\mathrm{SiO}_{2}$. J. Am. Ceram. Soc. 64, 360 (1981)

40. G. Yang, S. Cook, R.J. Hand, G. Möbus, $\mathrm{CeO}_{2}$ nano-precipitation in borosilicate glasses: a redox study using EELS. J. Euro. Ceram. Soc. 30, 831 (2010)

41. M.M. Sabri, G. Möbus, Electron irradiation induced nanocrystal formation in Cu-borosilicate glass. J. Nanopart. Res. 18, 1 (2016)

42. T. Gnanavel, M.A. Mat Yajid, Z. Saghi, Y. Peng, B.J. Inkson, M.R.J. Gibbs, G. Möbus, Electron beam nanofabrication of ferromagnetic nanostructures in TEM. Appl. Phys. A 102, 205 (2011)

43. G. Möbus, M. Ojovan, S. Cook, J. Tsai, G. Yang, Nano-scale quasi-melting of alkaliborosilicate glasses under electron irradiation. J. Nucl. Mater. 396, 264 (2010) 